\title{
AN IMPLEMENTATION OF ENERGY EFFICIENCY BY IMPROVED AODV PROTOCOL IN WSN
}

\author{
ANIL MALGAYA \\ M. Tech Student (IT) \\ Patel college of Science and Technology Indore (m.p)
}

\begin{abstract}
Wireless Sensor Network is an ad hoc network. Each sensor is defined with limited energy. Wireless sensor node deployed monitor into the network to the environmental condition or physical such as sound, temperature, vibration at different location. Each node collected the information than transmit to the base station. The data is transfer and receiving over the network each sensor consume some energy, for sending and receiving data. how much energy spent in each transmission define the lifetime of the network. The protocol play important roll, which can minimize the delay while offering high energy efficiency and long span of network lifetime. One of such protocol is MAODV, it is based on the chain structure, every chain have only one cluster head[2], it is in charge with every note's receiving and sending messages to the neighbouring node who belong to this chain, the cluster head spent or consume large energy and the times of every round increasing. In MAODV, it take the advantage of sending data to it the closet neighbour, it save the battery for WSN and increase the lifetime of the network. The proposed work is about to select the next neighboring node reliably. For this it will combine some parameters like such as Distance, Residual Energy and Response time. The proposed system will increase the overall communication and increase the network life.
\end{abstract}

Keyword: WSN, AODV, energy, node, MAODV

\section{INTRODUCTION}

Wireless Sensor Networks [1], with the characteristics of low cost, low energy consumption, distributed and selforganization, the information perception have a brought revolution. The wireless sensor network is composed of hundreds of thousands of the sensor nodes. For conditions of surrounding environment it can be sense such as illumination, temperature, and humidity. Each sensor node collects data such as illumination, humidity, and temperature of the area.and also it can be deployed and transmits data to base station. In variable fields The wireless sensor network can be applied For example, monitoring to the hostile environments for the use of military applications, forest fires to detecting for

\author{
PROF. PRAKASH MISHRA \\ Department of IT \\ Patel college of Science and Technology Indore (m.p)
}

prevention of disasters, or to study the phenomenon of the typhoon for a variety of academic purposes. These sensor nodes form a network can be self-organize to form a network and can communicate with the help of wireless interfaces with each other. a Energy efficient self-organization and initialization protocols are developed in [3], [4]. Each node has transmit power control and an omnidirectional antenna, and therefore can adjust the area of coverage with its wireless transmission. Typically, to perform a high-level task in a sensor web sensor nodes collect audio, seismic, and other types of data and collaborate. For example, a sensor network can be used for detecting the presence of potential threats in a military conflict. For receiving and transmitting data Most of battery energy is consumed. If all sensor nodes transmit data directly to the BS, the furthest node from BS will die early. On the other hand, among sensor nodes transmitting data through multiple hops, node closest to the BS tends to die early, leaving some network areas completely unmonitored and causing network partition., it is necessary for communication protocols In order to maximize the lifetime of WSN to prolong sensor nodes' lifetime by minimizing transmission energy consumption, sending data via paths that can avoid low energy sensor nodes and minimizing the total transmission power.

\section{WIRELESS SENSOR NETWORK}

Figure.1 shows a typical schematic of a wireless sensor network (WSN). After the initial deployment (typically ad hoc), sensor nodes are responsible for self-organizing an appropriate network infrastructure, often with multi-hop connections between sensor nodes [5]. on-board. the sensors can start collecting seismic, acoustic, magnetic or infrared information using either event driven or continuous working modes for the environment, Location and positioning information can also be obtained through the global positioning system (GPS) or local positioning algorithms. With the help of appropriately processed This information can be gathered from across the network to construct a global view of the monitoring phenomena or object. The basic philosophy behind WSNs is that, while the capability of each individual 
sensor node is limited, the aggregate power of the entire network is sufficient for the required mission.

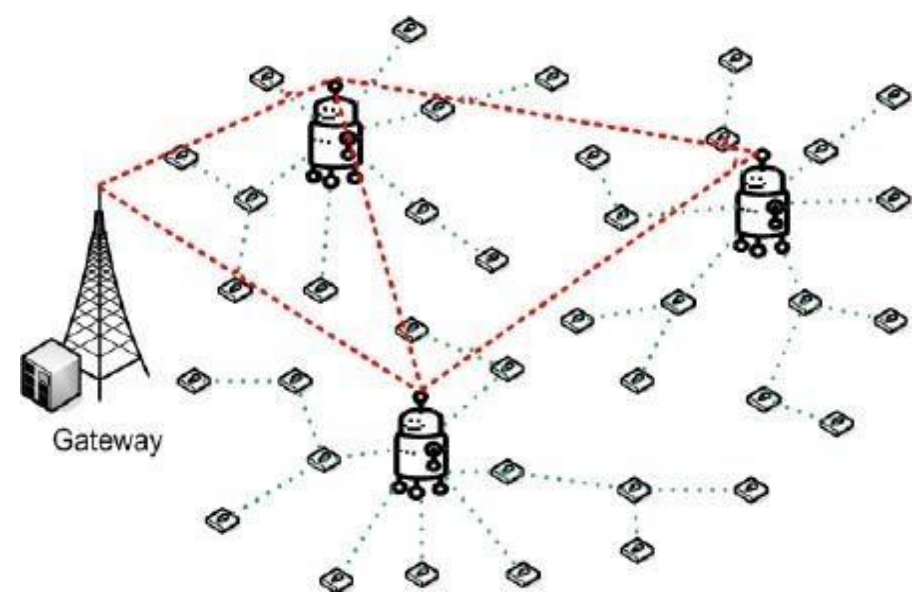

Fig 1 WSN Architecture

\section{PROPOSED ROUTING PROTOCOL}

\section{Improved AODV:}

The Improved AODV algorithm is based on the LEACH[15]. The core conception in MAODV is to form a chain among all the sensor nodes so that each node can receive from and transmit to the closest neighbor. Gathered data moves from node to node, get fused, and eventually a designated node (cluster head) transmits to the BS. for the average energy spent by each node per round is reduced so that Nodes take turns transmitting to the BS. The method of Building a chain to minimize the total length is similar to the traveling salesman problem, which is known to be intractable. However, with the radio communication energy parameters, a simple chain built with a greedy approach performs quite well. So, MAODV algorithm has some advantages as follow:

- Normal nodes only communicated with its neighbor and every node will take data fusion in order.

- The distance of the connect nodes with each other have been shortened remarkably.

- Nodes take turns to be the cluster head, so it takes no energy.

\section{IMPLEMENTATION AND RESULTS}

In this work, the random way point static model is used for the simulation of WSN routing protocols. The source-estimation pairs are spread randomly over the network where the point to point link is established between them. In this work UDP agent with CBR traffic is used with 40 packet size and 10kbps rate used for the transmission. The simulation configuration for static nodes consists of many network components and simulation parameters that are shown in the table in detail.

\section{NETWORK SIMULATION}

Generally network simulators try to model the real world networks. The principle idea is that if a system can be modelled, then future of the model can be changed and the corresponding results can be analysed. Following features are provided by simulator.

- Easy network topology setup

- Protocols and application implementation oUDP OFTP, Telnet, Web, CBR, VBR oRouting protocols oQueue management protocols

- Configurability

- Extensibility

\section{Packet Delivery Ratio:}

Packet delivery ratio is the ratio between the packets that are successfully delivered to a destination and the number of packets that have been sent by sender. The fig shows the effect to the packet delivery ratio (PDR) measured for the AODV, MAODV protocols when the node Density is increased.

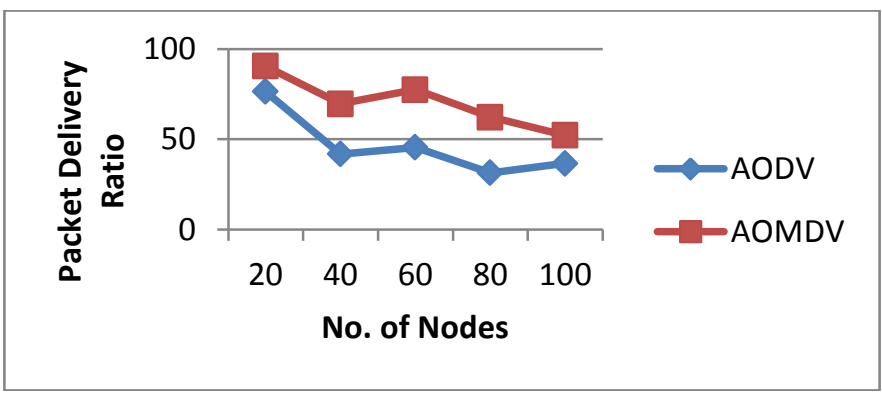

Fig: PDR Result

Throughput:

Network throughput in WSN communication channel is the average of successful message delivery over a communication. This data may be delivered over a logical or physical link, or pass through a certain network node. The throughput generally measured in bits per second or data packets per time slot.

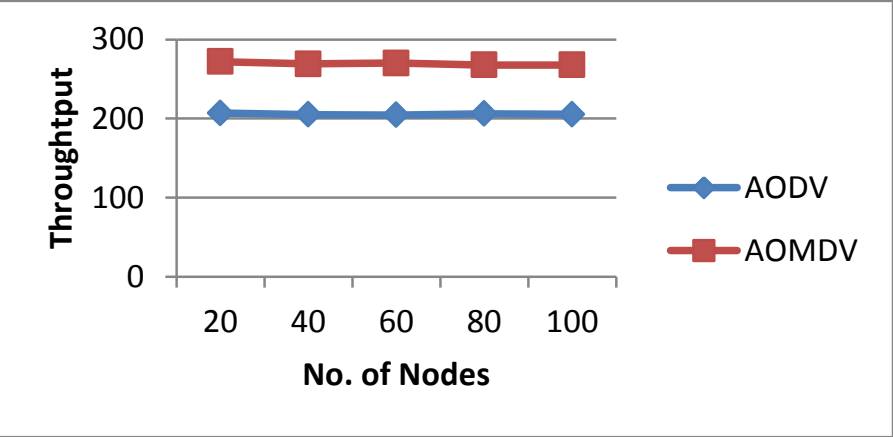

Fig: Throughput Result 


\section{International Journal of Engineering Applied Sciences and Technology, 2019 \\ Vol. 3, Issue 11, ISSN No. 2455-2143, Pages 67-70 \\ Published Online March 2019 in IJEAST (http://www.ijeast.com)}

\section{Energy:}

This is the average Energy between the sending of the data packet by the source and its receipt at the corresponding receiver. This includes all the delays caused during route buffering, acquisition, and processing at intermediate nodes.

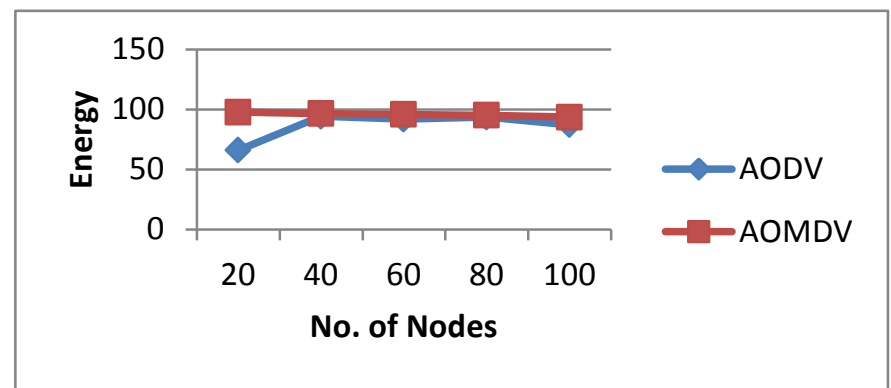

Fig: Energy Result

\section{End to End Delay:}

This is the average delay between the sending of the data packet by the source and its receipt at the corresponding receiver. This includes all the delays between the intermediate nodes caused during route acquisition, buffering and processing at.

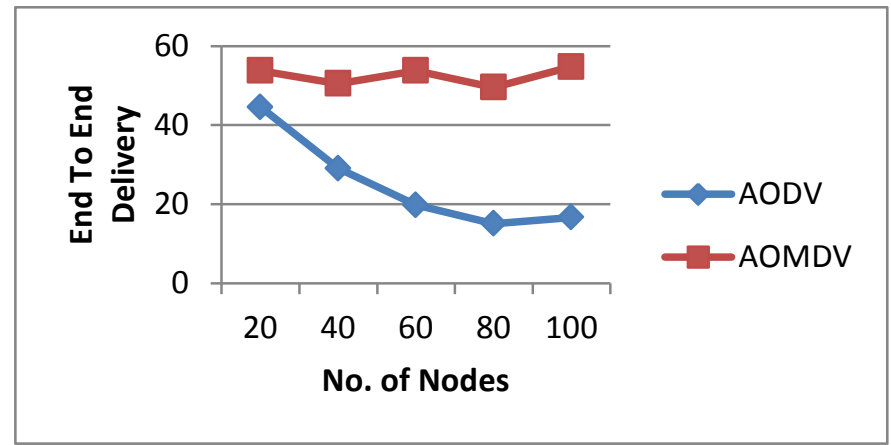

Fig: End To End Delay Result

\section{CONCLUSION}

In this paper, we describe AODV, it is chain based protocol that is near optimal for a data- gathering problem in sensor networks. AODV implementation NS2 by overhead of dynamic cluster formation are eliminating and minimizing the distance non leader-nodes fort transmitission, limiting the number of transmissions and receives among all nodes, and using transmission to the BS per round only ones. Due to the energy constraints, wireless sensors usually have a limited transmission range, making multi hop data routing towards the PN (processing node) more energy efficient than direct transmission (one hop). A primary design goal for wireless sensor networks is to use the energy efficiently. The proposed system will improve the existing AODV protocol. The simulation results shows that MAODV gives higher energy-efficiency in Network Simulation.

\section{REFERENCES}

[1] REN Feng-yuan, HUANG Hai-ning, and LIN Chuang, (2003) "Wireless Sensor Networks," Journal of Software, vol.14, (pp.1282-1291),

[2] CUI Li, Alekha Kumar Mishra and Manoj Kumar Samantara, (2016) "A Dynamic Energy- Efficient Chain Formation Scheme for AODV in Wireless Sensor Networks", International Conference on Computational Intelligence and Networks, IEEE2016.

[3] Feng Sen, Qi Bing, Tang Liangrui,(2011). An Improved Energy-Efficient AODV-Based Protocol in Wireless Sensor Networks, International Conference on Fuzzy Systems and Knowledge Discovery, vol.4, (pp.2230- 2233)

[4] W. Guo, W. Zhang, G. Lu,(2010). AODV Protocol in Wireless Sensor Network Based on an Improved Ant Colony Algorithm, IEEE Second International Workshop on Education Technology and Computer Science, Wuhan, (pp:64-67), March 2010.

[5] Sunita Rani, TarunGulati,(2012) An Improved AODV Protocol to Enhance Energy Utilization in Wireless Sensor Network, International Journal of Advanced Research in Computer Science and Software Engineering, vol.2, (pp.248-253),

[6] S. Lindsey, and CauligiS. Raghavendra,(2001) "AODV: Power-Efficient Gathering in Sensor Information Systems", 29 Sept 2001 IEEE, (pp. 11251130)

[7] Dali Wei, SerdarVural, Klaus Moessner,(2011) “An Energy-Efficient Clustering Solution for Wireless Sensor Networks" VOL. 10, NO.11,( pp.3973-3983),

[8] OssamaYounis, Sonia Fahmy, HEED(2004): A Hybrid, Energy-Efficient, Distributed Clustering Approach for Ad Hoc Sensor Networks, VOL. 3, NO. 4,(pp.366379).

[9] K. Nakano and S. Olariu,(2000) "Energy-Efficient Initialization Protocols for Single-Hop Radio Networks with No Collision Detection," IEEE Trans.Parallel and Distributed Systems vol. 11, no. 8,

[10] Ravinder Kumar, P. S. Mundra,(2012) Improved Data Gathering Protocol for WSN, International Journal of Electronics and Computer Science Engineering, vol.1, no.3, (pp.1208-1213) 2012.

[11] WANG Lin-ping, BI Wu, CAI Zhen and WANG Zufeng,(2010) Improved algorithm of AODV protocol 
introducing double cluster heads in wireless sensor network, International Conference on Computer, Mechatronics, Control and Electronic Engineering, (pp.148-151),

[12] K. Padmanabhan and P. Kamalakkannan,(2012) Energy-efficient Dynamic Clustering Protocol for Wireless Sensor Networks, International Journal of Computer Applications, Vol. 38, Issue. 11, (pp: 35-39)

[13] Dan Simon, Mehmet Ergezer, and Dawei Du,(2009) Population Distributions in Biogeography- Based Optimization Algorithms with Elitism, IEEE International Conference on Systems, Man and Cybernetics, (pp. 991 - 996).

[14] D.Simon,Biogeography-based optimization,(2008) IEEE Transactions on Evolutionary Computation, vol. 12, (pp. 702- 713).

[15] Rajudutta and Shishir Gupta,(2016) "Energy Aware Modified PEGASIES Through Packet Transmission in Wireless Sensor Network", Fourth International Conference on Parallel, Distributed and Grid Computing, 2016 IEEE. 